NETO, E. M. R.; MARQUES, L. A. R. V.; LOTIF, M. A. L.; LOBO, P. L. D.; MARTINIANO, C. R. Q.; FERREIRA, M. A. D.

REF - ISSN 1808-0804 Vol. XI (1), 48 - 60, 2014.

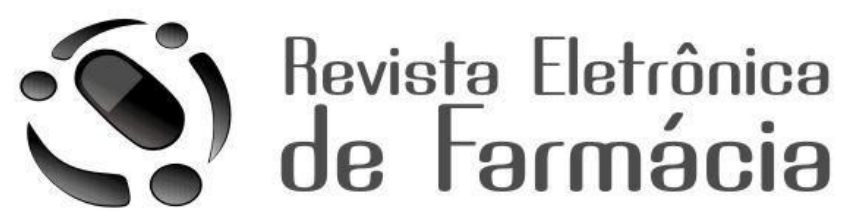

REF - ISSN 1808-0804 Vol. XI (1), 48 - 60, 2014.

\title{
TOXICIDADE DE ANESTÉSISCOS LOCAIS NA PRÁTICA CLÍNICA
}

\author{
LOCAL ANESTHETICS TOXICITY IN CLINICAL PRACTICE
}

TOXICIDAD DE LOS ANESTÉSICOS LOCALES EN LA PRÁCTICA CLÍNICA

\author{
Edilson Martins Rorigues Neto ${ }^{1}$, Lidia Audrey Rocha Valadas Marques ${ }^{2}$, Mara \\ Assef Leitão Lotif ${ }^{2}$, Patrícia Leal Dantas Lobo ${ }^{3}$, Carlos Ricardo de Queiroz \\ Martiniano ${ }^{4}$, Maria Augusta Drago Ferreira ${ }^{5}$.
}

\author{
${ }^{1}$ Mestrando do Programa de Pós-graduação em Farmacologia. Dep. Fisiologia e \\ Farmacologia. Universidade Federal do Ceará \\ ${ }^{2}$ Granduanda em Odontologia. Universidade Federal do Ceará \\ ${ }^{3}$ Professora do Curso de Odontologia - Sobral. Universidade Federal do Ceará \\ ${ }^{4}$ Professor do Curso de Odontologia. Dep. Clínica Odontológica. Universidade Federal do \\ Ceará \\ ${ }^{5}$ Professora do Curso de Farmácia. Dep. Farmácia. Universidade Federal do Ceará
}

Recebido em 08/01/2014, Aceito em 17/02/2014.

RESUMO: Anestésicos locais (ALS) são imprescindíveis a uma gama de procedimentos invasivos. Objetivou-se avaliar os diversos problemas sistêmicos devidos ao uso indistinto de ALs. Foram utilizadas as seguintes fontes: bases de dados eletrônicos tais como PUBMED, MEDLINE, LILACS e BBO, artigos em livros e periódicos, publicados entre os anos de 2005 a 2012. Foram usados os descritores: anestésicos locais, toxicidade, efeitos adversos e complicações sistêmicas. Dentre as complicações sistêmicas mais frequentes, foram relatados os efeitos no Sistema Nervoso Central (SNC) e Cardiovascular (SCV). Em níveis tóxicos de ALS no SNC a manifestação clínica primária é a convulsão tônico-clônica, podendo culminar em depressão generalizada de ambos os sistemas. Em nível de SCV, os ALs podem causar bradicardia e hipotensão arterial. Contrapondo os ALs, os vasoconstritores simpaticomiméticos geram efeitos excitatórios, que se intensificam ao se somar às catecolaminas endógenas. Podem ocorrer ainda reações de hipersensibilidade ou hematológicas. Anafilaxias verdadeiras ocorrem principalmente em ALs do tipo éster. Ademais, o metilparabeno e o bissulfito de sódio, incluídos no tubete de ALs, têm gerado sensibilização frequente. A alteração 
NETO, E. M. R.; MARQUES, L. A. R. V.; LOTIF, M. A. L.; LOBO, P. L. D.; MARTINIANO, C. R. Q.; FERREIRA, M. A. D.

REF - ISSN 1808-0804 Vol. XI (1), 48 - 60, 2014.

hematológica mais comum é a metemoglobinemia. Segundo a literatura, os ALs são fármacos seguros, mas é necessária perícia na técnica anestésica, adequação da dose e conhecimento sobre os cuidados necessários à sua utilização.

PALAVRAS-CHAVE: ANESTESIOLGIA; TOXICOLOGIA; FARMACOLOGIA CLÍNICA

ABSTRACT: Local anesthetics (LAs) are essential for most invasive procedures. This study aimed to evaluate the various systemic problems due to the indistinct use of LAs. Were used following sources: electronic databases such as PUBMED, MEDLINE, LILACS and BBO, articles in books and periodicals published between the years 2005 to 2012 . The descriptors were used: local anesthetics, toxicity, adverse effects and systemic complications. Among the most frequent systemic complications, the effects have been reported in the central nervous system (CNS) and cardiovascular (CVS). Toxic levels of LAs in the CNS is the clinical manifestation of primary generalized tonic-clonic seizures, and result in widespread depression of both systems. CVS-level, the LAs can cause bradycardia and hypotension. Opposing the LAs, sympathomimetic vasoconstictor generate excitatory effects, which intensify in joining the endogenous catecholamines. Can occur even hypersensitivity reactions or hematologic. True anaphylaxis occur mainly in LAs ester type. Furthermore, methylparaben and sodium bisulfite included in the cartridge of LAs are often generated sensitization. The most common hematological abnormality is methemoglobinemia. According to literature, the LAs are safe drugs, but it is necessary expertise in anesthetic technique, dose titration and knowledge about the care needed for their use.

KEY-WORDS: ANESTHESIOLOGY; TOXICOLOGY; CLINICAL PHARMACOLOGY

RESUMEN: Anestésicos locales (ALs) son esenciales para una amplia gama de procedimientos invasivos. Este estudio tiene como objetivo evaluar los diferentes problemas sistémicos debido al uso de fuerte regalamiento remitido ALs. Se utilizaron las siguientes fuentes: bases de datos electrónicas como PUBMED, MEDLINE, LILACS y BBO, artículos en revistas y libros, publicados entre los años de 2005 a 2012. Los descriptores se emplearon: anestésicos locales, toxicidad, efectos adversos y complicaciones sistémicas. Entre las complicaciones sistémicas más frecuentes, se informó sobre los efectos en el Sistema Nervioso Central (SNC) y cardiovascular (SCV). Los niveles tóxicos de als en el SNC manifestación clínica es la principal crisis convulsivas convulsiones tónico-clónicas generalizadas y pueden culminar en depresión generalizada de ambos sistemas. En el plano de la SCV, el ALS puede causar bradicardia e hipotensión. La contraposición esclerosis lateral amiotrófica, el vasoconstrictor simpaticomiméticos pueden generar efectos excitatorios, intensifican el resumen cuando las catecolaminas endógenas. Pueden producirse reacciones de hipersensibilidad o hematológicas. Pueden sufrir la anafilaxia verdadera ocurre principalmente en la esclerosis lateral amiotrófica tipo éster. Además, metil parabeno y bisulfito de sodio, están incluidos en las copas de la esclerosis lateral amiotrófica, ha generado conciencia frecuentes. La enmienda es el más común que afecta la metahemoglobinemia. De acuerdo con la literatura, la LAs son fármacos seguros, pero es necesaria la experiencia en la técnica anestésica, ajuste de la dosis y el conocimiento sobre los cuidados necesarios para su uso.

PALABRAS-CLAVE: ANESTESIOLOGÍA; TOXICOLOGÍA; FARMACOLOGÍA CLÍNICA

\section{INTRODUÇÃO}

A Anestesia local pode ser definida como um bloqueio, de modo reversível e transitório, da transmissão nervosa, que será determinado por uma perda das sensações em área delimitada do corpo sem alterações nos níveis de consciência. Desse modo, os Anestésicos locais ( $A L)$ são amplamente utilizados na prática clinica com a finalidade de 
NETO, E. M. R.; MARQUES, L. A. R. V.; LOTIF, M. A. L.; LOBO, P. L. D.; MARTINIANO, C. R. Q.; FERREIRA, M. A. D.

REF - ISSN 1808-0804 Vol. XI (1), 48 - 60, 2014.

eliminação da dor durante procedimentos invasivos. ${ }^{(1,2)}$

AL são bases fracas, apresentam pouca hidrossolubilidade e instabilidade em exposição ao ar, tendo pouco valor clínico nessa forma. No entanto, quando esse fármaco é combinado com um ácido, resulta na formação do sal anestésico, majoritariamente, na forma de cloridrato, desse modo, assume uma característica mais hidrossolúvel e estável. Os AL usados para infiltração são encontrados na forma do sal anestésico dissolvido em água destilada ou soro fisiológico. (3)

Em respeito à estrutura molecular os $\mathrm{AL}$ podem ser divididos em três grupos, com diferenças estruturais químicas: presença de um grupamento amina secundária ou terciária que the confere hidrossolubilidade. Um grupo aromático que aumenta o caráter apolar das moléculas e consequentemente sua lipofílicidade, que é essencial para a penetração nos neurônios transmissores. Finalmente, somando os dois grupamentos químicos, tem-se uma cadeia intermediária que apresentará caráter estrutural e propriedades físicoquímicas congruentes aos dois grupos anteriores, além de dois aspectos de fundamental importância: inicialmente o isolamento espacial necessário entre as extremidades lipofílica e hidrofílica, assim como a ligação química entre os dois grupamentos, servindo como base para a classificação dos anestésicos locais em dois grupos: os ésteres (-COO) e as amidas (-NHCO-). A cadeia intermediária é de grande relevância, já que há grandes diferenças no grau de alergenicidade, na potência e no metabolismo, quando realizada uma comparação entre os fármacos isolados. $(2-7)$

Uma anestesia local realizada com um produto de qualidade e técnica adequada culminará na abolição das funções autonômicas e sensitivomotoras. A inibição da transmissão em fibras periféricas obedece a uma sequencia já bem elucidada: bloqueio inicial das fibras autonômicas, responsáveis pela sensação térmica, tátil e dolorosa; seguidas pelas relacionadas à pressão e vibração e por fim as proprioceptivas e motoras. Essa sequencia depende do diâmetro, porção e da mielinização das fibras nervosas. A recuperação das funções nervosas se dá na ordem inversa. ${ }^{(1,4)}$

O agente anestésico ideal deve apresentar baixa toxicidade sistêmica; não ser irritante aos tecidos e também não causar lesão permanente às estruturas nervosas. O tempo para início da anestesia deve ser o mais curto possível e a duração de ação suficiente para a realização do procedimento invasivo, com ação reversível. (8)

Os $\mathrm{AL}$, de modo semelhante a outros fármacos não apresentam isenção de toxicidade. Os eventos mais comuns são a cardiotoxicidade e a 
NETO, E. M. R.; MARQUES, L. A. R. V.; LOTIF, M. A. L.; LOBO, P. L. D.; MARTINIANO, C. R. Q.; FERREIRA, M. A. D.

REF - ISSN 1808-0804 Vol. XI (1), 48 - 60, 2014.

neurotoxicidade. Desse modo torna-se essencial o conhecimento do profissional de saúde que utilize AL na sua pratica diária a noção dos riscos mais comuns e restrições necessárias inerentes ao seu uso. ${ }^{(4,9)}$

\section{METODOLOGIA}

Foi realizada uma revisão de literatura com pesquisa nos bancos de dados do Pubmed, Bireme, Lilacs e Scielo, bem como pesquisa nos periódicos fornecidos pelo Portal Brasileiro da Informação Científica sobre o assunto. Foram usados os descritores:

\section{REVISÃO DE LITERATURA}

Segundo a literatura, as reações sistêmicas de toxicidade mais frequentes decorrentes do uso de AL podem ser: Alérgicas; Cardiotoxicidade; Neurotoxicidade; Hematotoxicidade e Mistas. ${ }^{(7,9)}$

Eventos de toxicidade por $\mathrm{AL}$ em adultos podem estar sujeito a subnotificação, podendo ser classificados como muito raros, mas as estimativas sugerem cerca de 20 casos por 10.000 bloqueios de nervos periféricos e 1,2 para 10.000 anestesias epidurais, ocorrendo uma taxa de mortalidade em torno de 0,023 casos para 100.000 . $^{(9)}$

\section{ALÉRGICAS}


NETO, E. M. R.; MARQUES, L. A. R. V.; LOTIF, M. A. L.; LOBO, P. L. D.; MARTINIANO, C. R. Q.; FERREIRA, M. A. D.

REF - ISSN 1808-0804 Vol. XI (1), 48 - 60, 2014.

função farmacológica propriamente dita, mas serão necessários para garantir a estabilidade do produto final. Muitas dessas substâncias podem causar reações de hipersensibilidade tipo I. Recebendo especial interesse em relação às anafilaxias, estão os derivados do parabeno (metil, etil, proril e butilparabeno), incluídos na formulação do AL como um agente conservante para prevenir a proliferação bacteriana. Este grupo de compostos químicos pode gerar uma sensibilização contínua, pois sua aplicação não se restringe aos $A L$, podendo ser encontrado também em uma gama de formulações farmacêuticas e cosméticas. $(8,10,11)$

Outro agente potencialmente alergênico é látex, esse pode estar presentes nas extremidades do tubete do anestésico (stopper e diafragma no local de penetração da agulha). Desse modo pequenas quantidades do alérgeno podem penetrar durante $o$ procedimento anestésico, sendo suficiente para precipitar uma reação anafilática. ${ }^{(12)}$

O bissulfito de sódio, excipiente farmacêutico com função antioxidante para 0 agente vasoconstrictor em formulações anestésicas, é componente que tem causado frequentes reações anafiláticas. Asmáticos glicocorticoídedependentes, geralmente, apresentam hipersensibilidade a esse composto. Um evento de grande risco na pratica anestésica nesse grupo é a possibilidade de desenvolvimento de broncoespasmo severo. Um fato relevante sobre esse composto é a ausência de reação cruzada com antimicrobianos da classe das sulfonamidas. ${ }^{(4,6,8,12)}$

Em pacientes alérgicos ao bissulfito de sódio, pode-se utilizar de modo alternativo, AL sem a presença de vasoconstritores, pois assim se elimina a necessidade daquele composto na formulação. Nessas situações são opções terapêuticas a mepivacaina $3 \%$ e Prilocaina 4\% ambas na forma de Cloridrato. Esses anestésicos apresentaram atividade vasoconstritora, o que aumenta o tempo de ação e dispensa a necessidade de adição de epinefrina na sua fórmula $e$, consequentemente, do bissulfito de sódio. ${ }^{(8,12)}$

Alguns pacientes podem declarar que sofrem de alergia a agentes vasoconstritores, mas na verdade, foi observado que apresentam sinais e sintomas relacionados ao nível aumentado de catecolaminas plasmáticas, tais como palpitações, arritmias e taquicardia, ocasionadas pelo aumento dos níveis de ansiedade no período de pré-procedimento anestésico. (12)

Reações alérgicas locais podem ocorrem com o uso de anestésicos tópicos (benzocaína e tetracaína), utilizados principalmente na préanestesia local em odontologia e durante procedimentos de endoscopia. Como não 
NETO, E. M. R.; MARQUES, L. A. R. V.; LOTIF, M. A. L.; LOBO, P. L. D.; MARTINIANO, C. R. Q.; FERREIRA, M. A. D.

REF - ISSN 1808-0804 Vol. XI (1), 48 - 60, 2014.

há absorção desses fármacos a reação é limitada à área de aplicação. ${ }^{(12)}$

\section{CARDIOTOXICIDADE}

Atualmente existe dificuldade em se avaliar a frequência da toxicidade no sistema cardiovascular dos anestésicos locais devido à falta de estudos em grande escala, assim como baseados em evidências; porem mesmo com a escassez de estudos pode-se citar uma taxa teórica de 0,98 reações por 1.000 procedimentos. ${ }^{(9)}$

A cardiotoxicidade é comumente atribuída ao rápido aumento das concentrações plasmáticas ou doses excessivamente altas dos $\mathrm{AL}$, decorrentes, geralmente, de uma técnica anestésica insatisfatória, na qual o fármaco atinge a circulação sistemica. Esse evento segue uma via bifásica de ação: em concentrações mais baixas, ocorre à ativação do sistema nervoso simpático, durante essa fase excitatória o sistema nervoso central (SNC) pode conduzir eventos de hipertensão e taquicardia. Isto pode mascarar os efeitos depressores diretos no miocárdio, que ocorrem com concentrações mais elevadas dos $A L$, caracterizadas por arritmias ventriculares, atrasos na condução do miocárdio e disfunção contrátil profunda levando a colapso cardiovascular. ${ }^{(13)}$

O principal mecanismo de toxicidade cardíaca refere-se ao bloqueio dos canais miocárdicos de sódio voltagem-dependente, o que gera uma diminuição da duração do intervalo da onda PR e provoca um prolongamento, dose-dependente, do tempo de condução na QRS; assim como uma eventual depressão da atividade marca-passo. Um bloqueio dos canais de sódio persistente irá predispor a arritmias recorrentes. Este evento eletrofisiológico é agravado pelo efeito inotrópico negativo direto comum aos $A L$, que gerará bradicardia e hipotensão. O bloqueio de os canais de potássio e de cálcio podem também contribuir para cardiotoxicidade, o que significa até três sítios de ação para os AL. (7)

Muitas vezes, os AL estão associados, em sua formulação, com agentes vasoconstritores, estes pode ser simpaticomimético ou análogo da vasopressina. Os agentes sintéticos simpatomiméticos e catecolaminas endógenas podem causar ativação simpática em receptores $\beta_{1}$ do coração, culminando em taquicardia, palpitações, arritmias e aumento do débito cardíaco; e nos receptores $a_{1}$ dos vasos sanguíneos elevação da pressão arterial por vasoconstricção. Tendo em vista que numa técnica anestésica ideal não ocorra absorção sistêmica do agente vasoconstritor, Esses efeitos são mais comuns em pacientes ansiosos, nos quais a concentração de epinefrina e norepinefrina endógenas é aumentada 
NETO, E. M. R.; MARQUES, L. A. R. V.; LOTIF, M. A. L.; LOBO, P. L. D.; MARTINIANO, C. R. Q.; FERREIRA, M. A. D.

REF - ISSN 1808-0804 Vol. XI (1), 48 - 60, 2014.

cerca de 20 a 40 vezes durante o procedimento. ${ }^{(14)}$

O paciente hipertenso merece especial atenção ao utilizar um anestésico local com vasoconstritor, dados publicados sugerem que a utilização dessa associação durante procedimentos invasivos é importante, pois reduz a dor e aumenta o conforto; mas é fundamental reduzir a ansiedade do paciente durante $\mathrm{o}$ ato clínico ou cirúrgico para que não haja o aumento da pressão arterial, por stress. Com a finalidade de obter-se uma margem de segurança devem-se respeitar as doses limites de administração dos anestésicos com vasoconstrictor. A associação anestésica composta por vasoconstrictores simpaticomiméticos (norepinefrina ou levonoderfrina) deve ser evitada, já que estes ativam unanimemente receptores $a_{1}$, o que pode resultar em aumento exarcebado da pressão arterial. Outras contraindicações aos AL contendo vasoconstritores incluem a hipertensão grave não-controlada, arritmias, infarto do miocárdio ou acidente vascular cerebral no prazo de seis meses, angina coronária instável dentro de 3 meses, insuficiência cardíaca congestiva nãocontrolada, hipertireoidismo descontrolado. ${ }^{(14,15)}$

Outro fator preocupante é a ocorrência de fibrilação atrial isolada após a administração de $\mathrm{AL}$ com vasoconstrictor. Esse efeito ocorre devido ao aumento da contratilidade do miocárdio, sendo resultado da estimulação de receptores a cardíacos após a associação entre catecolaminas endógenas e exógenas em altas doses, como se verifica em situações de stress. (15)

Um fato interessante é a existência de uma correlação entre a lipossolubilidade do $A L$ e inibição da contractilidade cardíaca, essa evidência adicional, clinicamente relevante, permite concluir que a ropivacaína é menos tóxica do que a levobupivacaína, que, por sua vez, é menos tóxica que a bupivacaína. ${ }^{(7)}$

\section{NEUROLóGICOS}

As reações do tipo neurológicas são consideradas raras sendo citada na literatura uma taxa de 1 a 4 eventos por 1.000 bloqueios. ${ }^{(9)}$

Os $A L$, por conta de suas características físico-químicas, atravessam facilmente a barreira hematoencefálica. No SNC iram desempenhar uma ação depressora. Em concentrações séricas baixas (terapêuticas, não-tóxicas) não ocorrem efeitos clinicamente significantes no SNC, mas em doses tóxicas ou superdosagem a manifestação clínica primária é a convulsão tônico-clônica generalizada. ${ }^{(8)}$ 
NETO, E. M. R.; MARQUES, L. A. R. V.; LOTIF, M. A. L.; LOBO, P. L. D.; MARTINIANO, C. R. Q.; FERREIRA, M. A. D.

REF - ISSN 1808-0804 Vol. XI (1), 48 - 60, 2014.

A amígdala parece ser o principal foco neurofisiológico para convulsões, enquanto o hipocampo é posto como um foco secundário. (8) A posição da amígdala, situada no polo do lobo temporal abaixo do córtex no lado medial, parece ser um elemento crítico nos circuitos do cérebro que processam medo e agressão, desse modo pode-se associar a neurotoxicidade dos AL com alterações emocionais agudas no paciente. ${ }^{(16)}$

A Neurotoxicidade central está relacionada especificamente com as concentrações de AL no SNC e do seu efeito sobre o complexo de interações entre as vias excitatórias e inibitórias da neurotransmissão. Inicialmente, existe uma fase generalizada de excitação manifestada por atividade convulsiva. Esta fase inicial parece ser o resultado de bloqueio da via inibitória na amígdala, que permite que os neurônios excitatórios funcionem sem oposição; o possível sítio de ligação pode ser 0 receptor gabaérgico nos canais de cloreto. (16) Desse modo, em concentrações plasmáticas mais elevadas, a função dos neurônios inibidores é completamente deprimida, permitindo que os neurônios exitatórios funcionem sem oposição. O impulso de facilitação sem inibição produz a convulsão tônico-clônica observada nesses casos. ${ }^{(8)}$

$\mathrm{Na}$ ocorrência de um aumento considerável dos níveis séricos, o AL leva a uma depressão das vias de exitação e inibição, produzindo uma depressão generalizada do SNC, consequentemente, quedas na pressão arterial, frequência cardíaca e respiratória. ${ }^{(8)}$

Efeitos adversos sistêmicos geralmente decorrem de concentrações plasmáticas elevadas, resultantes de superdosagem, absorção rápida a partir de sítios periféricos ou injeção intravascular acidental, afetando a fisiologia do SNC, coração e circulação periférica. ${ }^{(4)}$

Tendo em vista evitar a administração de doses acima da máxima recomenda o profissional deve realizar o cálculo da dose por massa corporal ou ter noção, pelo menos, aproximada, do volume máximo; principalmente em grupos de pacientes diferenciados como na pediatria e geriatria. Nesses primeiros, durante procedimentos invasivos na cabeça e região oral, o risco de toxicidade é maior devido ao seu menor peso corporal, que não é representado proporcionalmente pela dimensão da anatomia orofacial. Enquanto o peso da criança pode ser cerca de um terço do peso do adulto, esta proporção não ocorre com o tamanho dos maxilares. Isto leva a uma falsa necessidade de administração de doses maiores do que as recomendadas. Nos idosos deve-se levar em consideração o seu metabolismo 
NETO, E. M. R.; MARQUES, L. A. R. V.; LOTIF, M. A. L.; LOBO, P. L. D.; MARTINIANO, C. R. Q.; FERREIRA, M. A. D.

REF - ISSN 1808-0804 Vol. XI (1), 48 - 60, 2014.

diminuído, o que repercutirá numa menor depuração do fármaco. ${ }^{(17)}$

Tabela 1 - Anestésicos locais nas concentrações comumente empregadas no Brasil, e suas doses máximas para adultos saudáveis. Adaptado de Motan et al., 2007(17)

\begin{tabular}{ccc}
\hline $\begin{array}{c}\text { Anestésico } \\
\text { Local }\end{array}$ & $\begin{array}{c}\text { Dose máxima (por kg de } \\
\text { peso corporal) }\end{array}$ & $\begin{array}{c}\text { Máximo absoluto } \\
\text { (independente do peso) }\end{array}$ \\
\hline Lidocaína 2\% & $4,4 \mathrm{mg}$ & $300 \mathrm{mg}$ \\
Mepivacaína 2\% & $4,4 \mathrm{mg}$ & $300 \mathrm{mg}$ \\
Mepivacaína 3\% & $4,4 \mathrm{mg}$ & $300 \mathrm{mg}$ \\
Prilocaína 3\% & $6 \mathrm{mg}$ & $400 \mathrm{mg}$ \\
Articaína 4\% & $5 \mathrm{mg}$ & $500 \mathrm{mg}$ \\
Bupivacaína 0,5\% & $1,3 \mathrm{mg}$ & $90 \mathrm{mg}$ \\
\hline
\end{tabular}

Figura 01 - Relação entre doses de lidocaína, etidocaína e bupivacaína que causam respostas tóxicas do SNC e doses que produzem colapso cardiovascular. Adaptado de Dillane, 2010. (7)

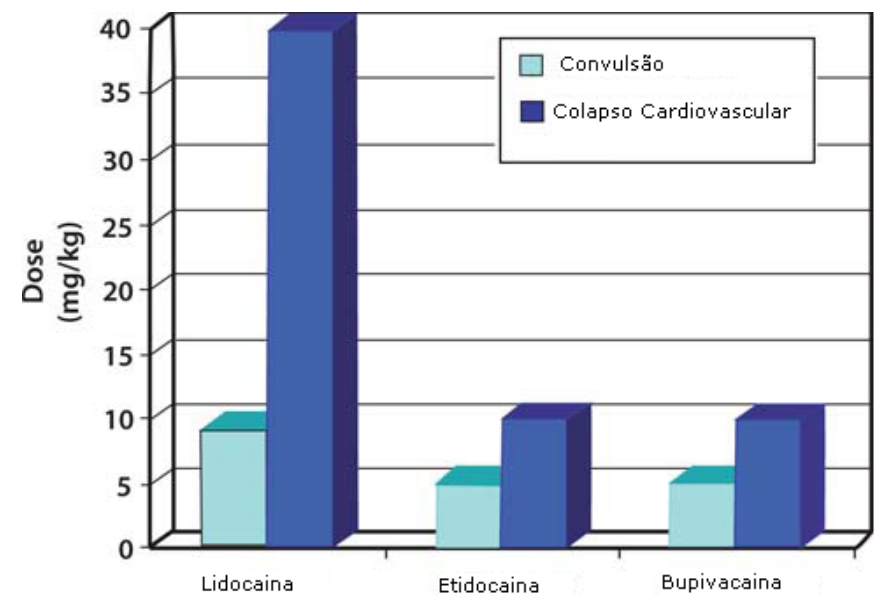

\section{HEMATOTOXICIDADE}

Uma complicação rara e potencialmente letal atribuída aos AL é a metemoglobinemia aguda, essa condição exige diagnóstico precoce e tratamento rápido. Nessa situação patológica, o ferro do grupo heme da hemoglobina é oxidado da forma de íon ferroso a íon férrico pelo metabólito ortotoluidina, desse modo ocorre 0 bloqueio do transporte do oxigênio pelos eritrócitos. 
NETO, E. M. R.; MARQUES, L. A. R. V.; LOTIF, M. A. L.; LOBO, P. L. D.; MARTINIANO, C. R. Q.; FERREIRA, M. A. D.

REF - ISSN 1808-0804 Vol. XI (1), 48 - 60, 2014.

Esse efeito adverso é associado comumente ao uso de prilocaína, mas que também pode ocorrer com articaína e aplicação tópica de benzocaína. ${ }^{(4,11)}$

Em níveis baixos de metemoglobinemia a cianose é primeiro sinal a ser manifestado seguido, em ordem gradual do aumento da relação metemoglobina/hemoglobina, por náuseas, sedação, convulsões e num desfecho mais grave o coma. A Metemoglobinemia é mais comum em neonatos devido à menor resistência da hemoglobina fetal a estresse oxidativo e imaturidade de enzimas que convertem ferro do seu estado férrico novamente ao estado ferroso (deficiência relativa de metemoglobina redutase em eritrócitos). Essa reação adversa é revertida por si só dentro de poucas horas em indivíduos sadios, à medida que o fármaco e seus metabólitos são eliminados. ${ }^{(2)}$

O emprego de baixas doses de prilocaína é considerado seguro em indivíduos sadios, porém esta não deve ser administrada a pacientes com qualquer condição associada à oxigenação deficiente, como hemoglobinopatias e outras anemias. Recomenda-se uma dose total máxima de prilocaína de $600 \mathrm{mg}$ em pacientes normais. ${ }^{(4)}$

Uma revisão de efeitos adversos relatados a Food and Drug Administration (FDA) sugeriu que ocorreram mais de 132 casos de metemoglobinemia induzida por benzocaína no período de 1997 e 2002, dos quais dois casos foram fatais. A discussão comum relatada à FDA foi o uso de múltiplas aplicações de sprays de benzocaína ou uma aplicação por um período acima do recomendado. ${ }^{(18)}$

\section{AÇÕES MISTAS}

Os AL apresentam uma grande complexidade farmacológica, atuando em diversos sítios de ação. Além de agir bloqueando a transmissão nociceptiva nos neurônios, também podem realizar bloqueio neuromuscular. Essa ação normalmente é discreta e em geral clinicamente insignificante. Entretanto, em algumas ocasiões especiais ela pode se adicionar à ação produzida por relaxantes musculares despolarizantes (succinilcolina) e não-despolarizantes (atracúrio, vecurônio), causando períodos anormalmente prolongados de paralisia muscular. ${ }^{(1)}$

Depressores do SNC (opióides, ansiolíticos, fenotiazinas e barbitúricos), geralmente, quando administrados concomitantemente a $\mathrm{AL}$, levam à potencialização das suas ações depressoras sobre o SNC. ${ }^{(9)}$

\section{CONSIDERAÇÕES FINAIS}

A anestesia local é essencial para a realização de uma gama de procedimentos invasivos, logo é extremamente importante que o 
NETO, E. M. R.; MARQUES, L. A. R. V.; LOTIF, M. A. L.; LOBO, P. L. D.; MARTINIANO, C. R. Q.; FERREIRA, M. A. D.

REF - ISSN 1808-0804 Vol. XI (1), 48 - 60, 2014.

profissional tenha familiaridade com a solução anestésica que será injetada, especialmente, em relação a sua ação e contra-indicações; além de conhecer a técnica anestésica e adequar a dose de acordo com o peso corporal. Todos esses fatores colaboram para a prevenção de efeitos adversos sistêmicos.

\section{REFERÊNCIAS}

1. Paiva LCA, Cavalcanti AL. Anestésicos Locais em Odontologia: uma revisão de literatura. Rev Biol Saúde 2005; 11 (2): 35-42.

2. Adams V, Marley J, McCarrol C. Prilocaine induced methaemoglobinaemia in a medically compromised patient. Was this an inevitable consequence of the dose administered? Brit Dent J 2007; 203 (10): 585-7.

3. Chioca LR, Segura RCF, Andreatini R, Losso EM. Antidepressivos e anestésicos locais: interações medicamentosas de interesse odontológico. Rev Sul-Bras Odontol. 2010 Oct-Dec;7(4):466-73

4. Brunton LL, Chabner BA, Knollmann BC. Anestésicos Locais. In: As Bases Farmacológicas da Terapêutica de Goodman \& Gilman. 12a ed. São Paulo: Artmed; 2012. p. 331-6.

5. Wannmacher L, Ferreira MBC. Anestésicos Locais. In: Wannmacher L, Ferreira MBC. Farmacologia Clínica Para Dentistas. $3^{a}$ ed. Rio de Janeiro: Guanabara Koogan; 2007. p.154-78

6. Yagiela JA. Anestésicos Locais. In: Yagiela JA, Dowd FJ, Johnson BS, et al. Farmacologia e Terapêutica para Dentistas. $6^{a}$ ed. Rio de Janeiro: Elsevier; 2012. p. 24371.

7. Dillane D, Finucane BT. Local anesthetic systemic toxicity. Can J Anesth/J Can Anesth (2010) 57:368-380

8. Malamed, S. F. Manual de anestesia local . 6. ed. Rio de Janeiro: Elsevier. 2013

9. Gómez REV, Araque HFG. Toxicity Due to Local Anesthetic Agents: Literature Review. Rev Col Anest 2011; 39 (1): 40-54

10. Moore PA, Hersh EV. Local Anesthetics: Pharmacology and Toxicity. Dent Clin N Am 54 (2010); 587-99

11. Becker DE, Reed KL. Local Anesthetics: Review of Pharmacological Considerations. Am Dent Soc Anest 2012; 59:90-102

12. Speca SJ, Boyne SG, Cuddy MA. Allergic Reactions to Local Anesthetic Formulations. Dent Clin N Am 2010; 54: 655-64.

13. Brown D. Local anesthetic toxicity. In: Finucane BT, editor. Complications of Regional Anesthesia. New York: Springer;2007. p. 61-73

14. Manani $G$, et al. Isolated atrial fibrillation (IAF) after local anaesthesia with epinephrine in an anxious dental patient. Br Dent J 2008; 205 (10): 539-41. 
NETO, E. M. R.; MARQUES, L. A. R. V.; LOTIF, M. A. L.; LOBO, P. L. D.; MARTINIANO, C. R. Q.; FERREIRA, M. A. D.

REF - ISSN 1808-0804 Vol. XI (1), 48 - 60, 2014.

15. Elad $\mathrm{S}$, et al. The cardiovascular effect of local anesthesia with articaine plus $1: 200,000$ adrenalin versus lidocaine plus $1: 100,000$ adrenalin in medically compromised cardiac patients: a prospective, randomized, double blinded study .Oral Surg Oral Med Oral Pathol Oral Radiol Endod 2008; 105 (6): 725-30

16. Bear MF, Connors BW, Paradiso MA. Brain Mechanisms of Emotion. Neuroscience Exploring the Brain. Philadelphia: Lippincott Williams \& Wilkins; 2007. p. 572

17. Motan MF, Cogo K, Bergamaschi CC, Volpato MC, Andrade ED. Mortalidade relacionada ao uso de anestésicos locais em odontologia. Rev Gauc Odont, 2007; v. 55 (2), 197-202.

18. Gutta, et al. Methemoglobinemia - an unusual cause of intraoperative Hypoxia. Oral Surg Oral Med Oral Pathol Oral Radiol Endod 2007; 103 (2): 197-202. 
NETO, E. M. R.; MARQUES, L. A. R. V.; LOTIF, M. A. L.; LOBO, P. L. D.; MARTINIANO, C. R. Q.; FERREIRA, M. A. D.

REF - ISSN 1808-0804 Vol. XI (1), 48 - 60, 2014. 